\title{
Unreliable Sensor Grids: Coverage, Connectivity and Diameter
}

\author{
Sanjay Shakkottai \\ Department of Electrical and \\ Computer Engineering \\ University of Texas at Austin \\ Email: shakkott@ece.utexas.edu
}

\author{
R. Srikant \\ Department of General Engineering \\ University of Illinois \\ at Urbana-Champaign \\ Email: rsrikant@uiuc.edu
}

\author{
Ness Shroff \\ Department of Electrical and \\ Computer Engineering \\ Purdue University \\ Email: shroff@ecn.purdue.edu
}

\begin{abstract}
We consider an unreliable wireless sensor gridnetwork with $n$ nodes placed in a square of unit area. We are interested in the coverage of the region and the connectivity of the network. We first show that the necessary and sufficient conditions for the random grid network to cover the unit square region as well as ensure that the active nodes are connected are of the form $p(n) r^{2}(n) \sim \frac{\log (n)}{n}$, where $r(n)$ is the transmission radius of each node and $p(n)$ is the probability that a node is "active" (not failed). This result indicates that, when $n$ is large, even if each node is highly unreliable and the transmission power is small, we can still maintain connectivity with coverage.

We also show that the diameter of the random grid (i.e., the maximum number of hops required to travel from any active node to another) is of the order $\sqrt{n / \log (n)}$. Finally, we derive a sufficient condition for connectivity of the active nodes (without necessarily having coverage). If the node success probability $p(n)$ is small enough, we show that connectivity does not imply coverage.

methods keywords: Stochastic processes/Queueing theory
\end{abstract}

\section{IntRoduction And System Model}

Recently, there has been a surge of interest in large-scale sensor networks. It is expected that these networks will be deployed for various applications, ranging from military applications (for intrusion detection) to civilian applications, such as smart buildings, where sensors could handle various aspects ranging from monitoring structural stability to monitoring the air quality and ventilation. Each node in such a network is expected to have very simple processing and sensing capability, a low-power source, and a low-power radio transmitter and receiver. The deployment of a large network of such nodes has become feasible primarily due to the availability of cheap wireless technology, and the emergence of microsensors based on MEMS technology [1], [2].

Some sensor networks can be viewed as examples of ad hoc packet radio networks. Capacity and scaling laws for large ad hoc networks have been obtained in [3], [4], [5]. Distributed, robust algorithms for routing in such networks have been studied in [6], [7]. For work on routing, MAC, coverage, location identification, etc. that are specific to sensor networks, we refer the reader to [8], [9], [10], [11].

In this paper, we are concerned with sensor networks that are inherently unreliable. We consider a network with $n$ nodes, arranged in a grid over a square region of unit area, as shown in Figure 1. The separation between adjacent nodes is $1 / \sqrt{n}$

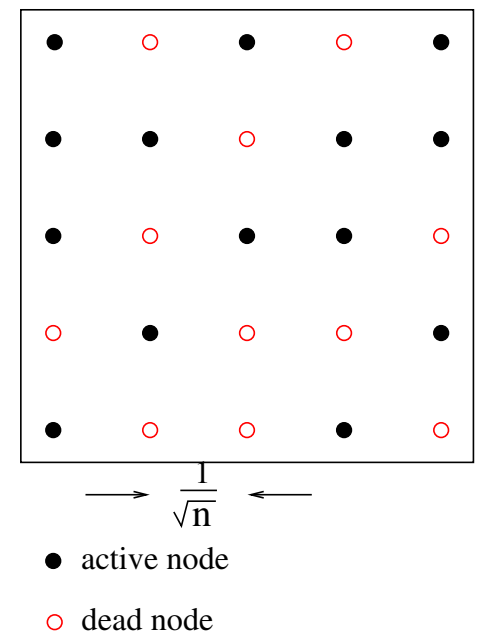

Fig. 1. A grid network with unreliable nodes

units. Each node is a sensor, and can detect events within some distance from it, called the sensing radius. The nodes are failure-prone: each node fails independently with probability $1-p(n)$. Thus, each node is active with probability $p(n)$. A pair of nodes can also communicate with each other if the distance between them is less than some specified value. For example, the bound on the communicating distance can be due to power constraints at each node. We let $r(n)$ denote the transmission/sensing radius of a node. Thus, two active nodes can communicate directly with each other only if they are at a distance less than or equal to $r(n)$ from each other. For notational convenience, we assume in this paper that the sensing radius is the same as the transmission radius. From the results and arguments presented in later sections, it will be clear that our results can easily be extended to the case where the sensing radius is different form the transmission radius. We will show the dependence of the quantities $p(n)$ and $r(n)$ on the size of the network and develop scaling laws for these quantities.

Two important performance metrics for such a network are connectivity and coverage. We say that the network is connected if any active node can communicate with any other active node (possibly using other active nodes are relays). The 
unit square is said to be covered if every point in the square is within a distance $r(n)$ of an active node. As an example, these two performance metrics are important in the problem of detecting any intrusion in the region, and communicating to the suitable authority (fusion center). Coverage will ensure that the intrusion is detected and connectivity will ensure that messages are propagated to the appropriate authority (see [8]).

A related model that has been studied in literature [3] is to consider random placement of nodes over a unit area. While a random approach may be necessary for certain cases, when possible, it would be preferable to actually place the nodes in particular locations. A natural placement strategy over a unit square is the grid layout, which is what we consider in this paper. If we have random placement of reliable nodes (i.e., nodes that don't fail), the authors in [3] have shown that as as long as the transmission radius of each node is of order $\sqrt{\log (n) / n}$, the network will remain connected. When we have uniform placement of reliable nodes (as in our case), it is clear that we need the transmission radius to scale only as $\sqrt{1 / n}$ for connectivity. In our case, the fundamental difference is that randomness arises in the network because of node failure. We are interested in scaling laws in this regime. One of our main results is that the network remains connected and covered if

$$
\sqrt{p(n)} r(n) \sim \sqrt{\frac{\log (n)}{n}} .
$$

The above results show that, interestingly, failure induced randomness leads to a similar scaling as randomness due to placement.

The arguments used in our proofs are quite different from those used in the random placement case. The arguments for connectivity of the network for the random placement case such as in [3] use percolation results for planar Poisson placement of nodes over an infinite plane such as in [12], [13]. The authors use clever arguments to relate this to the random placement problem by using the fact that conditional spatial Poisson processes lead to planar uniform distributions. However, for deterministic placement, as in our case, such arguments do not seem to work. We develop proofs based on "packing" space with circular bins. Further, the proofs we derive are constructive in nature, and lead to bounds on the diameter of the network.

\section{A. Main Results}

Our main contributions in this paper are:

(i) We show that the necessary and sufficient conditions for the random grid network to cover the unit square region as well as to ensure that the active nodes are connected are of the form

$$
p(n) r^{2}(n) \sim \frac{\log (n)}{n},
$$

where $p(n)$ is the node success probability and $r(n)$ is the transmission radius. This result indicates that, when $n$ is large, each node can be highly unreliable (i.e., $p(n)$ is small) and the transmission power can be small (i.e., $r(n)$ is small), and we can still maintain connectivity with coverage. In the process of obtaining this result, we also obtain upper and lower bounds on the probability of coverage and connectivity for finite $n$.

(ii) We show that the diameter of the random grid (i.e., the maximum number of hops required to travel from any active node to another) is of the order $\sqrt{n / \log (n)}$.

(iii) We derive a sufficient condition for connectivity of the active nodes (without necessarily having coverage). If the node success probability $p(n)$ is small enough, we show that connectivity does not imply coverage.

\section{B. Possible Applications}

The results obtained in this paper can be used to answer questions that arise in various applications. For example, a sensor field can be arranged in a grid and could be used for intrusion detection [14]. Since we expect to use a large number of nodes, it is unreasonable to assume that they will all be highly reliable, i.e., we expect node failures to occur relatively frequently.

In this context, some questions that can be answered using our results are:

(i): How small can the footprint of each node be so that connectivity can be maintained, and what is the trade-off between reliability and power expenditure? The radius of each node is a function of the power available at the node and the propagation model for the environment in which the node is placed. Thus, if a channel model is available, our results help in understanding the trade-off between reliability and power.

(ii): What is the resource usage of routing protocols in such a network? Many well-known routing protocols use flooding to perform route discovery between a source and destination, see [6] and other routing protocols in [7]. To control the dissemination of information using flooding, it is useful to provide a maximum number of hops over which flooding should take place. Our diameter results provide such an upper bound. Even for other routing strategies that are specific to sensor networks, such as the one presented in [9], where both the source and sink of a particular piece of information initiate directed routing queries, the diameter of the network would give a rough idea of the number of intermediate nodes that participate in the routing procedure. In [15], the authors study a geographical routing strategy for GPS equipped sensor nodes. They derive "worst-case" bounds to show that each node requires memory at most on the order of $\log (n)$ for storing routing tables for the geographical routing strategy to work. In the context of an unreliable GPS enabled grid network, we will later show that for $n$ large enough, the geographical routing strategy will ensure correct routing with no routing tables in any of the nodes, and we can provide bounds on the number of hops required. Finally we note that our proof of the diameter result will show that minimum-hop routing approximately mimics Euclidean shortest-path routing in our network. 
(iii): Suppose that we have a failure model for the individual nodes as a function of time. For example, it seems reasonable to expect that the probability of failure increases to 1 , i.e., each node becomes increasingly unreliable, as time progresses. Then, given some small parameter $\epsilon$, an important question to ask is: what is the maximum length of time over which we can expect the network to provide coverage and be connected with probability greater than or equal to $1-\epsilon$ ? By using the time-varying value of the failure probability of a node in our expressions for the upper and lower bounds on the probability of coverage and connectivity, we can obtain an estimate of this probability.

\section{Related Literature}

Seminal work in the area of scaling laws for radio networks was reported in [3], [4]. In [3], the authors studied scaling laws for connectivity of nodes placed at random over a unit area. For the same random placement model, the authors also derived the data carrying capacity of the network [4] in terms of the number of bit-meters/second. Their main result was that the throughput-meters scaled as $\frac{1}{\sqrt{n \log (n)}}$ per node.

In [5], the authors studied the capacity of the network with $n$ mobile nodes, with the nodes moving randomly over the unit area. They showed that node mobility increases capacity of the network. The key idea was that the source node gives copies of the data to a few intermediate nodes which happen to wander close to it. If one of the intermediaries or the source gets close to the destination node, the data is transferred. They show that this strategy increases capacity, albeit with an increase in end-to-end delay. In [13], the authors study coverage problems when various shaped objects (in particular, circular discs) are dropped over an infinite plane. In [12], the author studies connectivity problems (percolation problems) for a random node-placement model (node placement points are according to a two-dimensional spatial Poisson process).

Another random network model is the Bernoulli graph that has been studied extensively in [16]. The Bernoulli graph model consists of a network with $n$ nodes with a link between each pair of nodes with some probability $p(n)$. The fundamental difference of this model from a radio-graph model in this paper (and the model in [3]) is that in a Bernoulli graph, one can traverse arbitrary large distances in a single hop as there is a small, but finite probability, of a link between any pair of nodes. However, in the radio-graph model, there is a deterministic bound on the distance that can be traveled in a single hop.

In the context of gossip networks, the authors in [17], [18] study scaling results for reliable communication (i.e., connectivity). They develop a self-organizing, peer-to-peer protocol which converges to support a gossip algorithm reliably. The models they use are also Bernoulli graph models, but with directed arcs.

The results for the various models discussed above do not seem to be directly applicable to our model and the techniques used there do not seem to directly extend to our model.

\section{Organization of this Paper}

In the rest of this paper, in Section II, we study necessary and sufficient conditions for coverage with connectivity. Then, in Section III, we derive upper and lower bounds on the diameter of the network. We then consider connectivity of the network (without necessarily having coverage) in Section IV. We will see that as the network becomes more and more unreliable, the sufficient conditions for connectivity become weaker than the necessary conditions for coverage, i.e., connectivity does not imply coverage. We then present some numerical results in Section $\mathrm{V}$ and finally conclude in Section VI.

\section{Coverage With CONNECTIVITY: NeCESSARY AND SUFFICIENT CONDITIONS}

In this section, we study necessary and sufficient conditions for the network to cover the unit area as well as remain connected.

We now prove necessary conditions for coverage (and hence, for coverage with connectivity).

Proposition 2.1: Consider the random grid network with $n$ nodes, with each node being active at time $t$ with probability $p(n)$. Let $P_{c}(n)$ be the probability that the network covers the unit square. Then, we have

$$
P_{c}(n) \leq \exp \left[-\frac{e^{-\theta(p(n)) p(n) \pi r^{2}(n) n}}{4 r^{2}(n)}\right]
$$

where $\theta(p(n))=-\log (1-p(n)) / p(n)$. Further, a necessary condition for asymptotic coverage, i.e., $\lim _{n \rightarrow \infty} P_{c}(n)=1$, is given by

$$
\lim \inf _{n \rightarrow \infty} \frac{\theta(p(n)) p(n) r^{2}(n) n}{\log (n)} \geq \frac{1}{\pi} .
$$

Finally, suppose $p(n) \rightarrow 0$ as $n \rightarrow \infty$. Then, a necessary condition for coverage is given by

$$
\lim \inf _{n \rightarrow \infty} \frac{n p(n) r^{2}(n)}{\log (n)} \geq \frac{1}{\pi} .
$$

Proof: We tile the unit area with disjoint circles of radius $r(n)$ as shown in Figure 2. Thus, there are $J=\frac{1}{4 r^{2}(n)}$ circles, labeled as shown in the figure. We observe that a necessary condition for coverage of the unit square is that there should be at least one active node in each circle (otherwise the center of a circle will not be covered). Let

$P_{d}(n)=\operatorname{Pr}$ (there is at least one active node in each circle)

Hence, $P_{c}(n) \leq P_{d}(n)$. Now, by construction, the circles are disjoint. Observe that in each circle of radius $r(n)$, there are at most $\pi r^{2}(n) n$ nodes (active or dead). Further, as each node is active with probability $p(n)$, independent of any other node, 


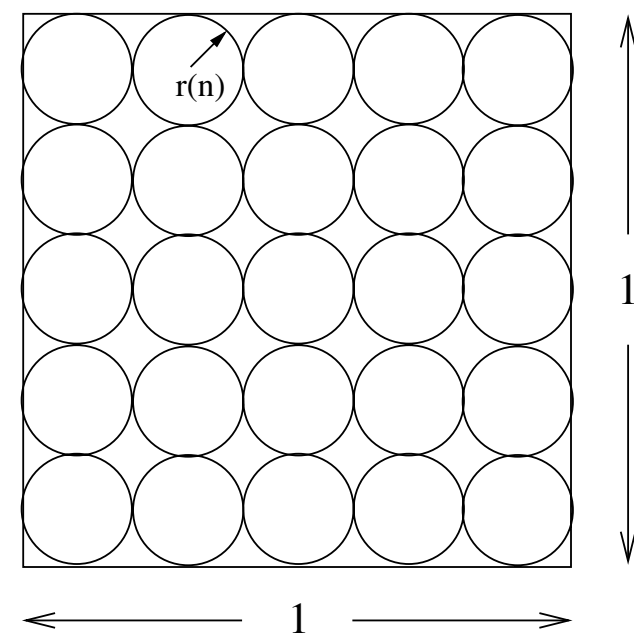

Fig. 2. Construction for a necessary condition for coverage

it follows that

$$
\begin{aligned}
P_{d}(n) & =\operatorname{Pr}\left(\bigcap_{j=1}^{J}\left\{\begin{array}{l}
\text { there is at least one } \\
\text { active node in circle } j
\end{array}\right\}\right) \\
& =[\operatorname{Pr}(\text { there is at least one active node in circle } 1)]^{J} \\
& \leq\left[1-(1-p(n))^{\pi r^{2}(n) n}\right]^{J} \\
& =\left[1-e^{-\theta(p(n)) p(n) \pi r^{2}(n) n}\right]^{\frac{1}{4 r^{2}(n)}} \\
& \leq \exp \left[-\frac{e^{-\theta(p(n)) p(n) \pi r^{2}(n) n}}{4 r^{2}(n)}\right]
\end{aligned}
$$

where $\theta(p)$ is defined in the statement of the theorem and we have used the fact that $1-p \leq e^{-p}$.

As we have $P_{c}(n) \leq P_{d}(n)$, to show (3), we need to find necessary conditions such that $P_{d}(n) \rightarrow 1$, as $n \rightarrow \infty$. It now follows from (4) that a necessary condition for $P_{d}(n) \rightarrow 1$, as $n \rightarrow \infty$, is given by

$$
4 r^{2}(n) e^{\theta(p(n)) p(n) \pi r^{2}(n) n} \stackrel{n \rightarrow \infty}{\rightarrow} \infty .
$$

We now define

$$
c(n)=\frac{n r^{2}(n)}{\log (n)} .
$$

Then, from (5), it follows that a necessary condition for $P_{d}(n) \rightarrow 1$ is given by

$$
4 c(n) \log (n) n^{c(n) \pi p(n) \theta(p(n))-1} \stackrel{n \rightarrow \infty}{\rightarrow} \infty .
$$

Now, assume that $p(n)$ satisfies $0 \leq \limsup _{n} p(n)<1$. $^{1}$ Then it is clear that if $c(n) \rightarrow 0$, then (7) does not hold since $\theta(p(n)) p(n)$ is bounded. Now consider the case where, for all $n$ large enough, $c(n) \geq c>0$. Then, (7) holds if and

\footnotetext{
${ }^{1}$ Note that the case $p(n) \rightarrow 1$ leads to the trivial necessary and sufficient condition that $r(n)=\frac{1}{\sqrt{n}}$ for coverage with connectivity.
}

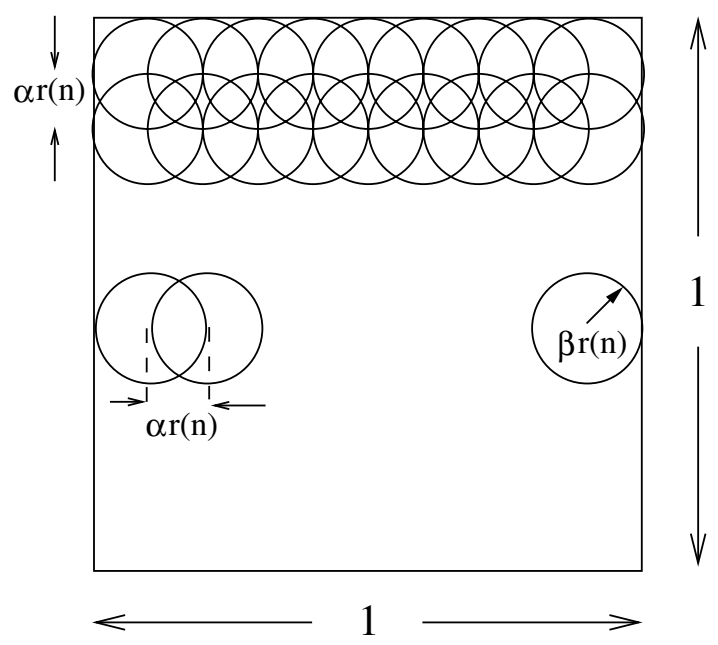

Fig. 3. Construction for a sufficient condition for coverage with connectivity

only if $c \pi p(n) \theta(p(n)) \geq 1$. Thus, a necessary condition for $P_{d}(n) \rightarrow 1$ is that, for all $n$ large enough,

$$
p(n) r^{2}(n) \geq \frac{1}{\pi \theta(p(n))} \frac{\log (n)}{n} .
$$

Suppose $p(n) \rightarrow p$ for some $0<p<1$. Then, condition (8) implies that a necessary condition for $P_{d}(n) \rightarrow 1$ is given by

$$
p(n) r^{2}(n) \geq \frac{1}{\pi \theta(p)} \frac{\log (n)}{n} .
$$

Finally, suppose $p(n) \rightarrow 0$, as $n \rightarrow \infty$. Fix any $\epsilon>0$. Then, it follows from the definition of $\theta(\cdot)$ that there exists $N_{0}$ such that for all $n \geq N_{0}, \theta(p(n)) \in[1,1+\epsilon]$. Thus, a necessary condition for coverage is that for all $n$ large enough,

$$
p(n) r^{2}(n) \geq \frac{1}{\pi(1+\epsilon)} \frac{\log (n)}{n} .
$$

Now because $\epsilon$ is arbitrary, the result follows.

Next, we prove sufficient conditions for coverage with connectivity.

Proposition 2.2: Consider the random grid network with $n$ nodes, with each node being active at time $t$ with probability $p(n)$. Then, for sufficiently large $n$,

$\operatorname{Pr}($ network is connected and covered $) \geq$

$$
1-\left(\frac{1}{\alpha r(n)}\right)^{2} e^{-p(n) \pi \beta^{2} r^{2}(n) n},
$$

for any $\alpha>0$ and $\beta>0$ such that $\alpha+2 \beta=1$. Further, a sufficient condition for asymptotic connectivity with coverage is given by

$$
\lim \inf _{n \rightarrow \infty} \frac{n p(n) r^{2}(n)}{\log (n)}>\frac{4}{\pi} .
$$




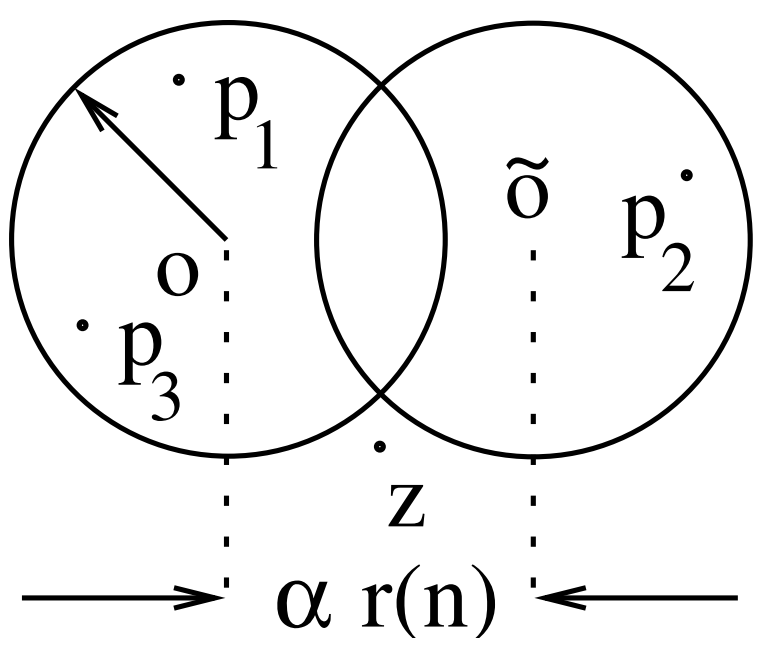

Fig. 4. Distances between active nodes: Coverage with connectivity

Proof: We cover the region of unit area with overlapping circles, each of radius $\beta r(n)$, and whose centers are separated by a distance $\alpha r(n)$. Here, $\alpha$ and $\beta$ satisfy

$$
\begin{aligned}
\alpha & >0, \\
\alpha+2 \beta & =1,
\end{aligned}
$$

as shown in Figure 3. We note that a sufficient condition for coverage with connectivity is that there is at least one active node in each circle (of radius $\beta r(n)$ ). To see this, consider active nodes $p_{1}, p_{2}, p_{3}$ and point $z$ as shown in Figure 4 , where $p_{1}, p_{3}$ are active nodes within a circle centered at a point $o, p_{2}$ is an active node in a neighboring circle centered at $\tilde{o}$ and $z$ is a point outside both circles. To show that this covering pattern does indeed ensure coverage with connectivity, it is sufficient to show that

$$
\begin{aligned}
d\left(p_{1}, p_{2}\right) & \leq r(n), \\
d\left(p_{1}, p_{3}\right) & \leq r(n), \\
d\left(p_{2}, z\right) & \leq r(n),
\end{aligned}
$$

where $d(.,$.$) is the Euclidean distance. Without loss of gener-$ ality, we can assume that $z$ is closer to point $\tilde{o}$ than point $o$. We then have

$$
\begin{aligned}
d\left(p_{2}, z\right) & \leq d\left(p_{2}, \tilde{o}\right)+d(\tilde{o}, z) \\
& \leq\left(\beta+\sqrt{\beta^{2}+\frac{\alpha^{2}}{4}}\right) r(n) .
\end{aligned}
$$

Now, we have $\alpha=1-2 \beta$. It also follows by construction that $\beta \leq 0.5$. Thus,

$$
\begin{aligned}
d\left(p_{2}, z\right) & \leq\left(\beta+\sqrt{\beta^{2}+(0.5-\beta)^{2}}\right) r(n) \\
& =(\beta+\sqrt{0.25-2 \beta(0.5-\beta)}) r(n) \\
& \leq(\beta+0.5) r(n) \\
& \leq r(n) .
\end{aligned}
$$

From the triangle inequality and the fact that $\beta \leq 0.5$, it trivially follows that $d\left(p_{1}, p_{3}\right) \leq r(n)$. Finally,

$$
\begin{aligned}
d\left(p_{1}, p_{2}\right) & \left.\leq d\left(p_{1} o\right)+d(o, \tilde{o})+d(\tilde{o}), p_{2}\right) \\
& \leq(\alpha+2 \beta) r(n) \leq r(n) .
\end{aligned}
$$

Now, we have $(1 /(\alpha r(n)))^{2}$ circles, each with area $\pi \beta^{2} r^{2}(n)$. Thus, the number of nodes (not necessarily active) in each circle is $\pi \beta^{2} r^{2}(n) n$.

We have neglected edge effects when counting the number of nodes in each circle. We have counted the number of nodes in each circle to be proportional to the area of the circle, whereas, the nodes are actually placed at discrete points with separation of $1 / \sqrt{n}$. However, it can be shown that this approximation is valid for $n$ large enough. We skip the details of this in this paper.

We now number the circles $1, \ldots,(1 /(\alpha r(n)))^{2}$ and define

$P_{s}=\operatorname{Pr}$ (there is at least one active node in each circle)

$\mathcal{A}_{i}=\{$ there is at least one active node in circle $i\}$

Our objective is to find sufficient conditions on $r(n), p(n)$ such that $P_{s} \rightarrow 1$ as $N \rightarrow \infty$. We now have

$$
\begin{aligned}
P_{s} & =\operatorname{Pr}\left(\bigcap_{i} \mathcal{A}_{i}\right) \\
& =1-\operatorname{Pr}\left(\bigcup_{i} \overline{\mathcal{A}}_{i}\right) \\
& \geq 1-\sum_{i} \operatorname{Pr}\left(\overline{\mathcal{A}}_{i}\right) \\
& =1-\left(\frac{1}{\alpha r(n)}\right)^{2}(1-p(n))^{\pi \beta^{2} r^{2}(n) n} \\
& \geq 1-\left(\frac{1}{\alpha r(n)}\right)^{2} e^{-p(n) \pi \beta^{2} r^{2}(n) n} .
\end{aligned}
$$

Thus, it follows that

$$
\left(\frac{1}{\alpha r(n)}\right)^{2} e^{-p(n) \pi \beta^{2} r^{2}(n) n} \stackrel{n \rightarrow \infty}{\rightarrow} 0 \quad \Longrightarrow \quad P_{s} \stackrel{n \rightarrow \infty}{\rightarrow} 1 .
$$

We now choose $r(n), p(n)$ such that for some $c>0$,

$$
p(n) r^{2}(n)>c \frac{\log (n)}{n} .
$$

We then have

$$
\left(\frac{1}{\alpha r(n)}\right)^{2} e^{-p(n) \pi \beta^{2} r^{2}(n) n}<\frac{1}{c \alpha^{2}} \frac{1}{\log (n)} \frac{1}{n^{\pi \beta^{2} c-1}} .
$$

It immediately follows that a sufficient condition for $P_{s} \rightarrow 1$ is

$$
c \geq \frac{1}{\pi \beta^{2}} .
$$

Also, it follows from construction that $\beta<0.5$. Thus, we have that

$$
r^{2}(n) p(n)>\frac{4}{\pi} \frac{\log (n)}{n}
$$




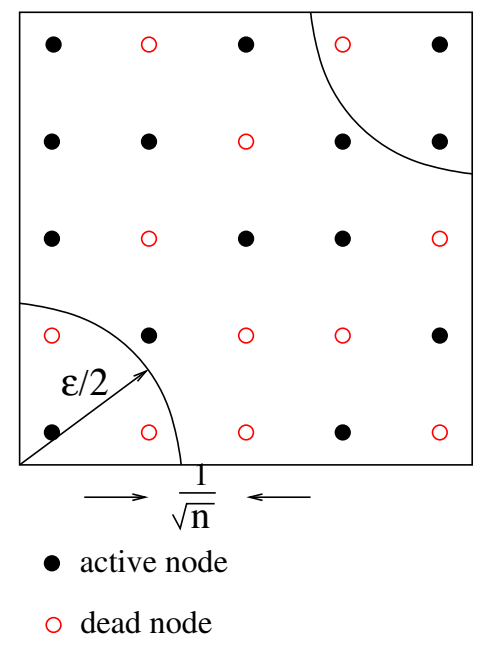

Fig. 5. Necessary condition for diameter of the grid

is sufficient for coverage with connectivity.

Remark 1: The results in this section provide us a means to answer the node replacement problem that was posed in Section I-B, Application (iii). Suppose that we are given a grid with $n$ nodes, and a system reliability parameter, i.e., the probability with which we desire the network to be connected and covered. Then, we can plot (1) and (9). For example, plots for a reliability of $95 \%$ and for different grid sizes are presented in Section V. Given a power budget, from these plots, we can provide an estimate of the minimum node-reliability $(p(n))$ for meeting our system-reliability objective. Now, if we have a node-reliability model versus time, we can immediately determine how long the network will remain connected and also provide coverage of the entire unit square.

\section{DiAMETER OF THE RANDOM GRID: UPPER AND LOWER BOUNDS}

As in [16], we define the diameter of the grid in the following manner: We label the active nodes $\{i=1,2, \ldots\}$ and let $D_{i j}(n)$ be the number of transmissions required to traverse from active node $i$ to active node $j$ (recall that each transmission traverses a distance of at most $r(n)$ ). Then, the diameter of the grid $D(n)$ is defined as

$$
D(n)=\max _{i, j} D_{i j}(n) .
$$

We next derive a lower bound on the diameter of the random grid by noting that, with high probability, there will be active nodes at diagonally opposite ends of the grid. Straight-line routing then provides the lower bound on the number of hops required to traverse from one end to the other.

Proposition 3.1: Suppose that $r(n), p(n)$ satisfy

Choose any $\epsilon>0$. Then, as $n \rightarrow \infty$, we have

$$
P(r(n) D(n)>\sqrt{2}-\epsilon) \rightarrow 1 .
$$

Proof: Consider two sectors at opposite ends of the square of unit area. The sectors are obtained by drawing arcs of radius $\epsilon / 2$ at diagonally opposite ends of the square of unit area as shown in Figure 5. We can easily show that if (2) is satisfied, then, as $n \rightarrow \infty$, we have that

$$
\operatorname{Pr}\left(\left\{\begin{array}{l}
\text { there is at least one active } \\
\text { node in each of the two sectors }
\end{array}\right\}\right) \rightarrow 1 .
$$

Now, as each transmission can traverse at most a distance of $r(n)$, and the diagonal distance from the edge sectors (see Figure 5) is $\sqrt{2}-\epsilon$, the result follows immediately from (12).

We now show an upper bound on the diameter of the random grid. The upper bound justifies the heuristic that the more power one is willing to use, the smaller the diameter of the grid.

Proposition 3.2: Suppose $p(n), r(n)$ satisfy

$$
p(n) r^{2}(n) \geq c \frac{\log (n)}{n},
$$

for some $c>\frac{4}{\pi}$. Then, as $n \rightarrow \infty$, we have

$$
P\left(D(n) r(n) \leq \frac{2}{1-\frac{2}{\sqrt{\pi c}}}\right) \rightarrow 1 .
$$

Proof: The result follows directly from the arguments in Proposition 2.2. Consider the construction in Figure 3, and fix some $\beta<0.5$ and $\alpha=1-2 \beta$. We define

$$
c=\frac{1}{\pi \beta^{2}} \text {. }
$$

From (10), we know that under these conditions, the network is connected and covered. It is easy to see from this construction that a routing strategy for traveling from any active node to another is simply to travel in the vertical direction from the source node till the $y$-coordinate of the destination node is reached, and then to travel in the $\mathrm{x}$-direction. In each hop, by construction, a distance of $\frac{1}{\alpha r(n)}$ is traversed. Thus, from (14), we have

$$
\alpha=1-\frac{2}{\sqrt{\pi c}} .
$$

The maximum distance that can be traversed on the grid is 2 (along the sides, from one end to the diagonally opposite end). Hence, the required result follows.

Remark 2: From (11) and (13), we have, for $n$ large enough,

$$
\sqrt{2}<r(n) D(n)<\frac{2}{1-\frac{2}{\sqrt{\pi c}}},
$$

where $c$ is the parameter that decides how much power each node uses or how reliable each node is.

The upper and lower bounds are of the same order and have the property that as $c$ increases, they become closer. Further, the constructive nature of the upper bound provides a heuristic for routing data over the grid. 
In each overlapping circle of Figure 3, one or more active nodes can be designated as relays, and the relays form an overlay network. Routing on this overlay network will lead to worst-case guarantees in the number of hops required to traverse the network.

Remark 3: It is interesting to note that, while the connectivity and coverage condition for the unreliable grid looks similar to the connectivity condition for Bernoulli graphs in [16], the diameter of the unreliable grid is much larger $(O(\sqrt{n p(n) / \log n}))$ than the diameter of Bernoulli graphs under critical connectivity (which is $O(\log (n))$, see [16]). This is due to the fact that, for every finite $n$, any pair of nodes in a Bernoulli graph can communicate with some non-zero probability, whereas, in our unreliable grid model, nodes can communicate only if they are within each others range.

Remark 4: As discussed earlier (in Application (ii) in Section I-B) in [15], the authors study the geographical routing strategy for GPS equipped randomly placed sensor nodes. Essentially, the geographical routing strategy is the following: At each hop, if there is an active node within the transmitting node's range that is closer to the destination than the transmitting node, the data packet is forwarded to that node. If not, a route discovery is initiated, and the routing entries are appropriately stored.

They derive "worst-case" bounds to show that each node requires memory at most on the order of $\log (n)$ for storing routing tables for the geographical routing strategy to work.

Now consider an unreliable GPS enabled grid network, and assume that Proposition 3.2 is satisfied. We pick any active source and destination node. It is clear from the construction of the bounds by means of overlapping circles that there will always be an active intermediate node within the coverage area of the source node such that the distance between the intermediate node and the destination node is smaller than the distance between the source and destination. Thus, it follows that, for $n$ large enough, no route discoveries need to be initiated. Thus, the geographical routing strategy will ensure correct routing with no routing tables in any of the nodes, and we can provide bounds on the number of hops required.

In fact, we can show that a similar construction as in Proposition 3.2 works even for the case of random placement of nodes. Thus, the necessity of routing tables is absent even if we randomly place nodes over a unit area. The details are available in a longer version of the paper [19].

\section{CONNECTIVITY OF THE UNRELIABLE GRID: SUFFICIENT CONDITIONS}

In this section, we derive sufficient conditions for connectivity of the active nodes. We first consider the case when every node in the network is active. In this case, it is clear that the conditions required for connectivity will ensure coverage of the unit square as well. At the other extreme, consider the

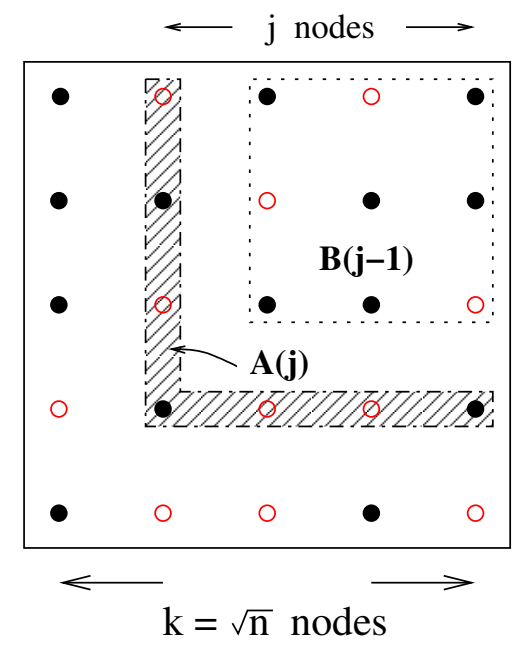

Fig. 6. Sub-grids and their boundaries used in the recursive argument for connectivity of active nodes

case when only one node is alive. Then, the network is trivially connected. However, in order to ensure coverage in this case, clearly the sensing radius has to be an order one quantity, i.e., the sensing radius should be at least 1 unit (does not scale with $n$ ).

We will explicitly show that, as the reliability of a node decreases, the sufficient condition for connectivity becomes weaker than the necessary condition for coverage with connectivity. Thus connectivity does not imply coverage.

Proposition 4.1: As before, we consider a grid with $n$ nodes. Then, for sufficiently large $n$,

$$
\operatorname{Pr}(\text { network is connected }) \geq 1-n p(n) e^{-\frac{\pi p(n) r^{2}(n) n}{2}} .
$$

Thus, the network is connected if $p(n)$ and $r(n)$ satisfy

$$
n p(n) e^{-\frac{\pi p(n) r^{2}(n) n}{2}} \stackrel{n \rightarrow \infty}{\rightarrow} 0 .
$$

Proof: For ease of exposition, we will assume that $\sqrt{n}$ is an integer, and let $k=\sqrt{n}$. Thus, we consider a grid with each side having $k$ nodes. When we refer to a sub-grid $S_{j}$ of size $j$, we refer to the $j^{2}$ nodes in the upper right-hand corner of the original grid. We use the notation $E_{j}$ to refer to the nodes on the bottom and left boundaries of $S_{j}$; see Figure 6 for an illustration of these definition. For each $j=1,2, \ldots, k$, we define the following events:

$$
\begin{gathered}
\mathcal{A}(j)=\left\{\begin{array}{l}
\text { at least one of the active nodes on the left } \\
\text { and bottom boundaries of the sub-grid } \\
\text { of side } j \text { is disconnected from } S_{j-1}
\end{array}\right\} \\
\mathcal{B}(j)=\{\text { The (sub)-grid of size } j \times j \text { is disconnected }\}
\end{gathered}
$$

We also define $q(l)$ to be the probability that the sub-grid $S_{l}$ is disconnected, i.e., $q(l)=\operatorname{Pr}(\mathcal{B}(l))$. Thus, by definition, $q(k)$ is the probability that the network is disconnected. We will derive sufficient conditions for $q(k) \rightarrow 0$ using a recursive 


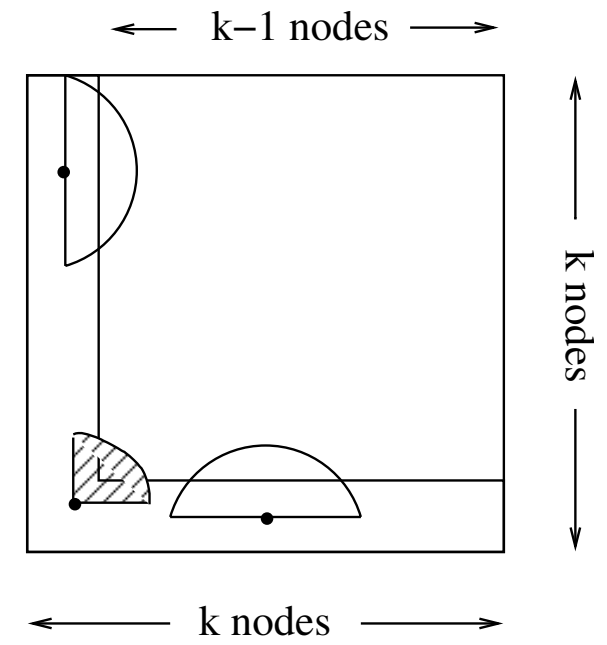

Fig. 7. Semi-circular regions to be examined for connectedness of boundary nodes

argument. First, we note that

$$
\begin{aligned}
q(j) & =\operatorname{Pr}(\mathcal{B}(j)) \\
& =\operatorname{Pr}(\mathcal{A}(j) \bigcup\{\overline{\mathcal{A}(j)} \bigcap \mathcal{B}(j-1)\}) \\
& \leq \operatorname{Pr}(\mathcal{A}(j))+\operatorname{Pr}(\overline{\mathcal{A}(j)} \bigcap \mathcal{B}(j-1)) \\
& =\operatorname{Pr}(\mathcal{A}(j))+\operatorname{Pr}(\overline{\mathcal{A}(j)} \mid \mathcal{B}(j-1)) \operatorname{Pr}(\mathcal{B}(j-1)) \\
& \leq \operatorname{Pr}(\mathcal{A}(j))+q(j-1)
\end{aligned}
$$

where we have used the notation $\overline{\mathcal{E}}$ to denote the complement of an event $\mathcal{E}$.

Let $j=k$ and suppose that every active node on the left (or bottom) boundary has an active node in the right (or top) semi-circular region of radius $r(n)$ (strictly within $S_{k-1}$; see Figure 7). Then, it follows that every node in the boundary region $E_{k}$ is connected to $S_{k-1}$. Therefore,

$$
\begin{aligned}
& \operatorname{Pr}(\mathcal{A}(k)) \\
\leq & \operatorname{Pr}\left(\left\{\begin{array}{l}
\text { every active boundary node } \\
\text { has at least one active } \\
\text { node as a right (or top) neighbor }
\end{array}\right\}\right) \\
= & \operatorname{Pr}\left(\left\{\begin{array}{l}
\text { there is some active boundary node } \\
\text { that does not have an active } \\
\text { node as a right (or top) neighbor }
\end{array}\right\}\right) \\
= & \operatorname{Pr}\left(\bigcup_{j=1}^{2 k-1}\left\{\begin{array}{l}
\text { boundary node } j \text { is active } \\
\text { and does not have an active } \\
\text { node as a right (or top) neighbor }
\end{array}\right\}\right) \\
\leq & (2 k-1) p(n)(1-p(n))^{\frac{\pi r^{2}(n) n}{2}} .
\end{aligned}
$$

In the last step, we have neglected the corner effect, i.e., we need to consider the event that the corner node is active, and it has no active neighbor in the quarter-sector (shaded region in Figure 7). Also, we have counted the number of nodes in the right/top semi-circle as $\frac{\pi r^{2}(n) n}{2}$, which counts the nodes along the edge as well. However, we can show that these approximations do not matter (as the nodes in the interior of the semi-circle are of order $n r^{2}(n)$, while the edge nodes are of order $\sqrt{n} r^{2}(n)$ ), but we skip the details here for ease of exposition. These details are available in a longer version of the paper [19]. A similar argument holds for each $j$ as well, although there is another corner effect for small $j$ due to fact that size of grid $S_{j}$ may be smaller than $r(n)$. Again, one can show that this does not matter asymptotically. Thus, from (17) and (18), it follows that

$$
q(k)-q(k-1) \leq(2 k-1) p(n)(1-p(n))^{\frac{\pi r^{2}(n) n}{2}} .
$$

Now, by noting the recursion

$$
q(k)-q(0)=\sum_{j=1}^{k}(q(j)-q(j-1)),
$$

and defining $q(0)=0$, we have from (19),

$$
\begin{aligned}
q(k) & \leq k^{2} p(n)(1-p(n))^{\frac{\pi r^{2}(n) n}{2}} \\
& =n p(n)(1-p(n))^{\frac{\pi r^{2}(n) n}{2}} \\
& \leq n p(n) e^{-\frac{\pi p(n) r^{2}(n) n}{2}}
\end{aligned}
$$

where the last step follows from the fact $1-p \leq e^{-p}$, which was also used in the proof of an earlier theorem. The desired result now immediately follows.

Using this result, we now discuss the requirements for connectivity without coverage, and compare with the requirements for coverage.

Corollary 4.1: Fix any $\gamma \in(0,1)$, and let

$$
p(n)=\frac{1}{n^{\gamma}} .
$$

Then, the network is connected if $p(n), r(n)$ satisfy

$$
p(n) r^{2}(n) \geq c \frac{\log (n)}{n},
$$

where $c$ satisfies

$$
c>\frac{2}{\pi}(1-\gamma) \text {. }
$$

Proof: Substituting (20) and (21) in (16), the network is connected if

$$
\frac{n}{n^{\gamma}} \frac{1}{n^{c \pi}} \rightarrow 0
$$

i.e.,

$$
\frac{1}{n^{\pi c / 2+\gamma-1}} \rightarrow 0,
$$

as $n \rightarrow \infty$. Equation (22) follows immediately from the above condition.

Remark 5: Choosing $\gamma=0.75$, from Corollary 4.1, it follows that a sufficient condition for coverage is

$$
p(n) r^{2}(n)>\frac{0.5}{\pi} \frac{\log (n)}{n} .
$$




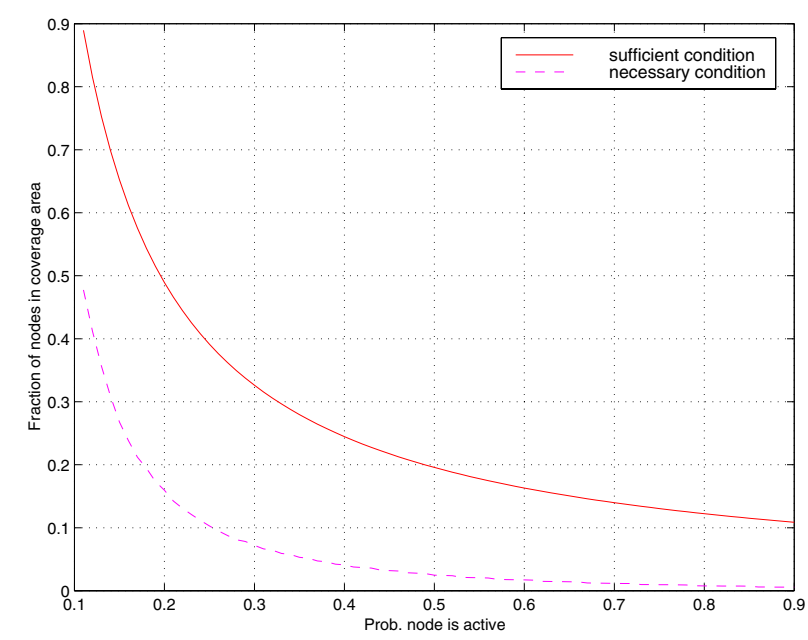

Fig. 8. Connectivity with coverage: Necessary and sufficient conditions. We have a grid network with $25 \times 25$ nodes.

On the other hand, from Proposition 2.1, a necessary condition for coverage is given by

$$
p(n) r^{2}(n) \geq \frac{1}{\pi} \frac{\log (n)}{n} .
$$

Thus, it is possible for the network to be connected, while not ensuring coverage of the unit square. In other words, connectivity does not imply coverage.

We finally observe in passing that the results in this section provide expressions which can be used to find the trade-off between power consumption and reliability while maintaining connectivity (posed in Application (i) in Section I-B).

\section{NumericAl Results AND Simulation}

In this section, we present numerical results for a random grid network. We consider a $25 \times 25$ grid as well as a $100 \times 100$ grid and graphically present the upper and lower bounds on the probability of coverage and connectivity (equations (1) and (9)).

In Figure 8, we consider a grid of $25 \times 25$ nodes. Our requirement is to ensure that coverage and connectivity are maintained with a probability of at least 0.95 . The figure shows the trade-off between the probability of node failure and how large the range of a single node has to be (normalized in terms of the fraction of the total number of nodes in the range of each node). For example, to ensure connectivity with coverage with the above probability, if each node fails with probability 0.5 , then the coverage radius $r(n)$ should be chosen such that that fraction of nodes in an active node's range is $20 \%$ of the whole region (unit square).

In Figure 9, a similar plot has been presented for a grid of $100 \times 100$. In this case, to maintain connectivity with coverage, each active node should be able to reach only $1.5 \%$ of the nodes (active or otherwise) if each node fails with probability 0.5 .

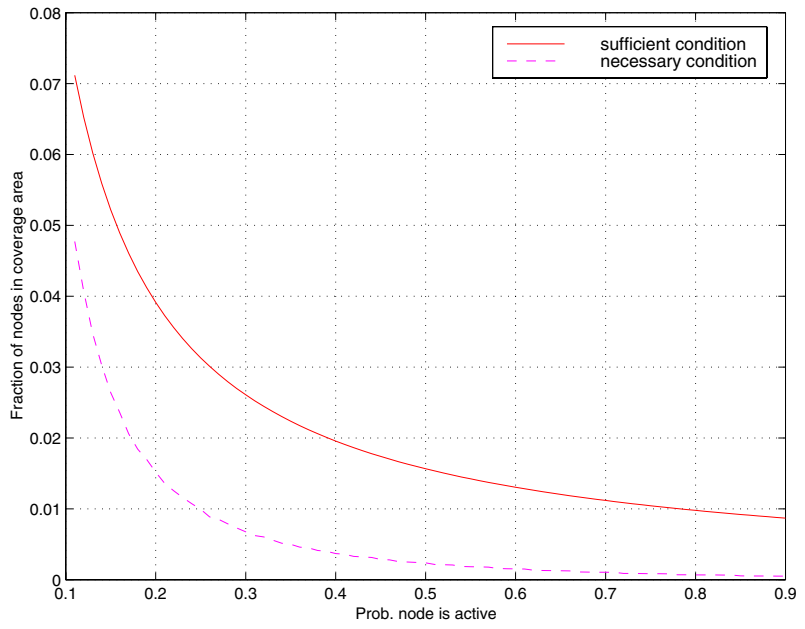

Fig. 9. Connectivity with coverage: Necessary and sufficient conditions. We have a grid network with $100 \times 100$ nodes.

Suppose that each node uses a transmission power $P(n)$, and suppose that for successful reception of data, a received power of at least $q$ is desired. Then, a simple model which relates the coverage radius $r(n)$ with these quantities is given by

$$
q=\frac{k P(n)}{[r(n)]^{\gamma}},
$$

for some $k>0$. Typical values of $\gamma$ for various propagation models [20] range from about 3 to 4 . Thus, $P(n)=$ $k^{\prime}\left(\pi r^{2}(n)\right)^{\gamma / 2}$. Using this model, we compare the total power required in the network for the case of the $25 \times 25$ grid and the $100 \times 100$ grid.

If we require the probability of coverage to be greater than 0.95 , as discussed earlier in this section, for the $25 \times 25$ grid, the total power required is $25 \cdot 25 \cdot k^{\prime}(0.2)^{\gamma / 2}$, whereas in the case of the $100 \times 100$ grid, the total power requirement is of the form $100 \cdot 100 \cdot k^{\prime}(0.015)^{\gamma / 2}$. Defining $\rho$ to be the ratio of the total power required in the $25 \times 25$ grid to that in the $100 \times 100$ grid, we find that for the propagation parameter $\gamma=3, \rho=3.05$, i.e., we need only one third of the total power to operate the larger grid with the same performance in terms of coverage and connectivity.

The intuitive reason for this result is that, as we scale up the number of nodes in the network, the power required per active node (and hence, the transmission radius) for connectivity and coverage decreases at a rate faster than the rate at which the number of nodes increase. Thus, to maintain coverage and connectivity, the total power required for the grid decreases as the number of nodes increase.

However, the penalty we pay for this is in the diameter of the network. From the results in Section III, numerical computations show that there is a 2.88 -fold increase in the diameter when we go from a $25 \times 25$ grid to a $100 \times 100$ grid. Thus, the speed of route discovery using schemes such as DSR or other schemes discussed earlier would roughly decrease by this factor. 


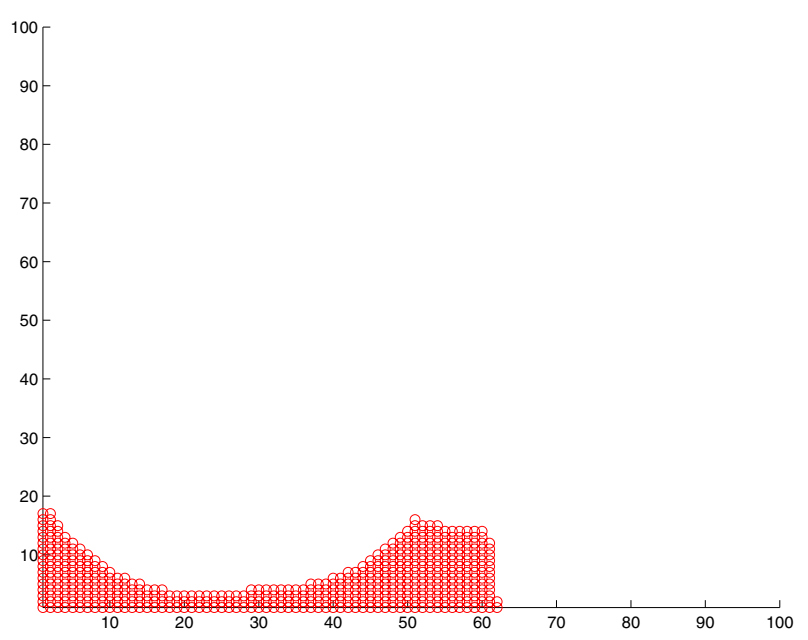

Fig. 10. Connectivity without Coverage: Simulation with a grid network with $100 \times 100$ nodes.

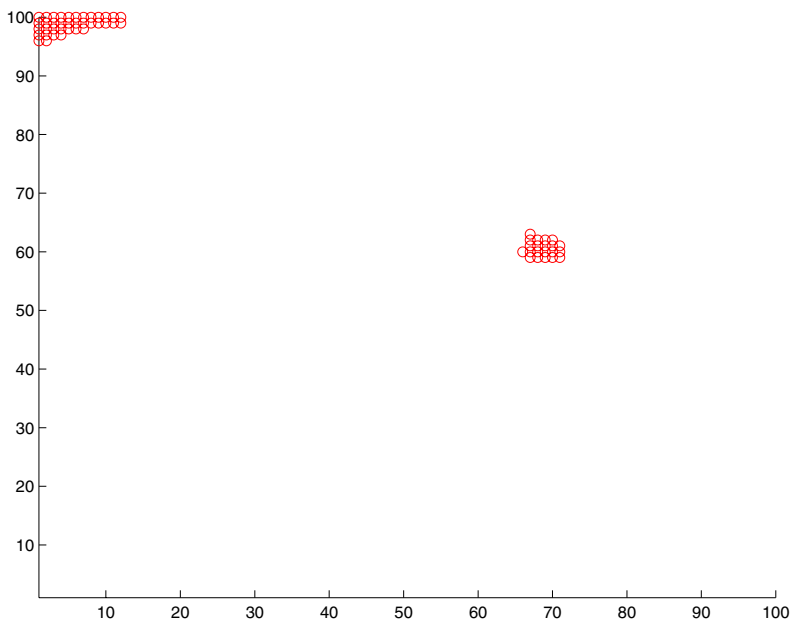

Fig. 11. Connectivity without Coverage: Simulation with a grid network with $100 \times 100$ nodes

Finally, recall that in Section IV, we showed that when the reliability of a node is small enough, connectivity does not imply coverage. In Figures 10 and 11, we have simulated a grid network of size $100 \times 100$ nodes. The plots show two realizations, with the reliability of each node $p(n)$ being $0.5 \%$, and each active node being able to reach $16 \%$ of the nodes (i.e., $\pi r^{2}(n)=0.16$ ). These conditions are sufficient for connectivity but not for coverage. In the figure, the shaded regions indicate uncovered regions, while the unshaded regions are covered. We can see that the unshaded regions in both plots are connected, thus indicating that we have connectivity but not coverage.

\section{CONCLUSION}

In this paper, we considered an unreliable wireless sensor grid-network with $n$ nodes placed over a unit area. Defining $r(n)$ as the transmitting radius of each node, and $p(n)$ as the probability that a node is active at some time $t$, we found that necessary and sufficient conditions for the grid network to cover the unit square region as well as ensure that the active nodes are connected are of the form $p(n) r^{2}(n) \sim \frac{\log (n)}{n}$. This result indicates that, when $n$ is large, each node can be highly unreliable and the transmission power can be small and we can still maintain connectivity with coverage.

We have also shown that the diameter of the random grid (i.e., the maximum number of hops required to travel from any active node to another) is of the order $\sqrt{n / \log (n)}$. A corollary of this is that the shortest-hop path between any pair of nodes is nearly the same as "straight-line" path between the nodes. Further, we have upper bounds on the number hops required.

Finally, we derived a sufficient condition for connectivity of the active nodes (without necessarily having coverage). If the node success probability $p(n)$ is small enough, we have shown that connectivity does not imply coverage.

\section{ACKNOWLEDGMENT}

This research has been supported by NSF Grants 9805441ANR, 0207728-ANR and DARPA grant F30602-00-2-0542.

\section{REFERENCES}

[1] J. M. Kahn, R. H. Katz, and K. S. J. Pister, "Mobile networking for smart dust," in Proceedings of MobiCom 99, (Seattle, WA), August 1999.

[2] K. Sohrabi, J. Gao, V. Ailawadhi, and G. Pottie, "Protocols for selforganization of a wireless sensor network," IEEE Personal Communications, vol. 7, pp. 16-27, October 2000.

[3] P. Gupta and P. R. Kumar, "Critical power for asymptotic connectivity in wireless networks," in Stochastic Analysis, Control, Optimization and Applications: A Volume in Honor of W.H. Fleming. Edited by W.M. McEneany, G. Yin, and Q. Zhang, (Boston), pp. 547-566, Birkhauser, 1998.

[4] P. Gupta and P. R. Kumar, "The capacity of wireless networks," IEEE Transactions on Information Theory, vol. IT-46, pp. 388-404, March 2000.

[5] M. Grossgluauser and D. Tse, "Mobility increases the capacity of ad-hoc wireless networks," in Proceedings of IEEE INFOCOM 2001, (Anchorage, Alaska), April 2001.

[6] D. Johnson, D. Maltz, and J. Broch, "DSR: The dynamic source routing protocol for multi-hop wireless ad hoc networks," in Ad Hoc Networking, edited by C. E. Perkins, pp. 139-172, Addison-Wesley, 2001.

[7] C. Perkins, Ad Hoc Networking. Addison-Wesley, 2001.

[8] S. Meguerdichian, F. Koushanfar, M. Potkonjak, and M. Srivastava, "Coverage problems in wireless ad-hoc sensor networks," in Proceedings of IEEE Infocom, (Anchorage, AK), 2001.

[9] D. Braginsky and D. Estrin, "Rumor routing algorithm for sensor networks," 2001. Available at http://lecs.cs.ucla.edu/estrin/.

[10] W. Ye, J. Heidemann, and D. Estrin, "An energy-efficient MAC protocol for wireless sensor networks," in Proceedings of IEEE Infocom, (New York, NY), June 2002.

[11] L. Doherty, L. E. Ghaoui, and K. S. J. Pister, "Convex position estimation in wireless sensor networks," in Proceedings of IEEE Infocom, (Anchorage, AK), April 2001.

[12] R. Meester and R. Roy, Continuum Percolation. Cambridge University Press, 1996

[13] P. Hall, Introduction to the Theory of Coverage Processes. John Wiley and Sons, 1988.

[14] "U.S. discusses giving India ground sensors," June 13 2002. News Item in Washington Post.

[15] R. Jain, A. Puri, and R. Sengupta, "Geographical routing using partial information for wireless ad hoc networks," IEEE Personal Communications, vol. 8, pp. 48-57, February 2001.

[16] B. Bollobas, Random Graphs. Academic Press, 1985.

[17] A.-M. Kermarrec, L. Massoulie, and A. Ganesh, "Reliable probabilistic communication in large-scale information dissemination systems," October 2000. Microsoft Research Tech Report MSR-TR-2000-105. 
[18] A.-M. Kermarrec, L. Massoulie, and A. Ganesh, "Scamp: Peer-to-peer lightweight membership service for large-scale group communication," in Proceedings of the Third International Workshop on Networked Group Communications (NGC 2001), (London, UK), November 2001.

[19] S. Shakkottai, R. Srikant, and N. B. Shroff, "Unreliable sensor grids: Coverage, connectivity and diameter," June 2002. CSL Technical Report.

[20] T. Rappaport, Wireless Communications: Principles and Practice. Prentice Hall, 2001. 